\title{
TOWARDS A WELL-DEFINED MEDIAN
}

\author{
AhMad Al-SALman AND MOWAFFAQ HaJJA
}

Abstract. The diagonal $\Delta$ of $\mathbb{R}^{n}$ is Chebeshev with respect to the $p$-norm for every $p \in(1, \infty]$ but not for $p=1$. As a result, the median is multi-valued, since the median of a data set $\left\{a_{1}, \cdots, a_{n}\right\}$ can be thought of as the number(s) $\mu$ for which the point $(\mu, \cdots, \mu)$ is a point on $\Delta$ that best approximates the point $\left(a_{1}, \cdots, a_{n}\right)$ with respect to the $\ell_{1}$-norm. In this note, it is proved that if $\left(\mu_{p}, \cdots, \mu_{p}\right)$ is the unique point on $\Delta$ that best approximates a fixed point $\left(a_{1}, \cdots, a_{n}\right)$ with respect to the $\ell_{p}$-norm for $p \in(1, \infty]$, then as $p$ decreases to $1, \mu_{p}$ converges, and its limit is proposed to be called the median of $\left\{a_{1}, \cdots, a_{n}\right\}$. Along the way, $\mu_{p}$ is shown to be continuous in $p$ for all $p \in(1, \infty]$ in the sense that $\mu_{p}$ converges to $\mu_{q}$ as $p$ goes to $q$ for every $q \in(0, \infty]$.

Mathematics subject classification (2000): 26E60, 41A50, 41A52.

Key words and phrases: Median, mean, best approximation, convex, Chebeshev set, Fermat-Torricelli point.

\section{REFERENCES}

[1] Z. ABu-ABbas, Private Communications, 1995.

[2] F. Galvin And S. D. Shore, Distance functions and topology, Amer. Math. Monthly, 98 (1991), 620-623.

[3] M. HAJJA, Distance means and best approximation means: Some elementary questions, preprint.

[4] Y. S. KUPITZ AND H. MARTINI, Geometric aspects of the generalized Fermat-Torricelli problem, Intuitive Geometry, Bolyai Society Mathematical Studies, Vol. 6, pp. 55-127, 1997. 